\title{
Intermittent glucocorticoid steroid dosing enhances muscle repair without eliciting muscle atrophy
}

\author{
Mattia Quattrocelli, David Y. Barefield, James L. Warner, Andy H. Vo, Michele Hadhazy, Judy U. Earley, \\ Alexis R. Demonbreun, and Elizabeth M. McNally
}

Center for Genetic Medicine, Northwestern University Feinberg School of Medicine, Chicago, Illinois, USA.

\begin{abstract}
Clucocorticoid steroids such as prednisone are prescribed for chronic muscle conditions such as Duchenne muscular dystrophy, where their use is associated with prolonged ambulation. The positive effects of chronic steroid treatment in muscular dystrophy are paradoxical because these steroids are also known to trigger muscle atrophy. Chronic steroid use usually involves once-daily dosing, although weekly dosing in children has been suggested for its reduced side effects on behavior. In this work, we tested steroid dosing in mice and found that a single pulse of glucocorticoid steroids improved sarcolemmal repair through increased expression of annexins A1 and A6, which mediate myofiber repair. This increased expression was dependent on glucocorticoid response elements upstream of annexins and was reinforced by the expression of forkhead box 01 (FOX01). We compared weekly versus daily steroid treatment in mouse models of acute muscle injury and in muscular dystrophy and determined that both regimens provided comparable benefits in terms of annexin gene expression and muscle repair. However, daily dosing activated atrophic pathways, including F-box protein 32 (Fbxo32), which encodes atrogin-1. Conversely, weekly steroid treatment in $\mathrm{mdx}$ mice improved muscle function and histopathology and concomitantly induced the ergogenic transcription factor Krüppel-like factor 15 (KIf15) while decreasing Fbxo32. These findings suggest that intermittent, rather than daily, glucocorticoid steroid regimen promotes sarcolemmal repair and muscle recovery from injury while limiting atrophic remodeling.
\end{abstract}

\section{Introduction}

Glucocorticoid (GC) steroids are used to treat many chronic conditions ranging from lung disease to autoimmune disorders and muscle disease. It is estimated that more than $1 \%$ of the US population is treated chronically with GC steroids. Although the side effect profile from GC steroids is extensive, muscle weakness and myopathy can occur in response to chronic GC steroid use and can be extremely debilitating (1). Curiously, chronic GC steroids are used to treat Duchenne muscular dystrophy (DMD), a primary muscle disease, and the use of steroids is associated with prolonged ambulation $(2,3)$.

The mechanisms underlying the beneficial effects of steroid use in muscular dystrophy are still largely unknown. In the US, the most commonly used agent is prednisone, while deflazacort is used in Europe and Asia. In muscular dystrophy, steroids are thought in part to act through immune system modulation, as a direct reduction in immune cell infiltration into muscle has been observed (4). However, GC steroids also directly affect muscle itself by shifting metabolism and gene expression (5). Chronic GC steroid use has been seen to reduce fibrosis in human DMD muscle (6). In $m d x$ mice, a genetic model of DMD, deflazacort was observed to improve muscle regeneration and growth after injury $(7,8)$. An 8 -week study of prednisolone in $m d x$ mice improved specific force

Conflict of interest: The authors have declared that no conflict of interest exists. Submitted: October 25, 2016; Accepted: March 9, 2017.

Reference information: / Clin Invest. 2017;127(6):2418-2432.

https://doi.org/10.1172/JCI91445 and decreased the number of centrally nucleated myofibers (9). However, 3 months of daily prednisone in $m d x$ mice resulted in weight loss, reduced strength, and increased fibrosis in the heart, suggesting that longer, chronic administration may be problematic (10). Recently, it has been shown that GC steroids rely on Krüppellike factor 15 (Klf15), a Krüppel like transcription factor, to mediate ergogenic muscle performance effects (11). Deletion of Klf15 in the mouse exacerbated the $m d x$ muscle phenotype, and muscle-specific overexpression of Klf15 improved muscle performance in the $m d x$ mouse. In addition, it is still unclear whether prednisone and deflazacort vary in their effects in DMD. Deflazacort is a synthetic oxazoline derivative of prednisolone. A recent comparative study in boys with DMD reported that deflazacort associated with higher efficacy, measured as later age at loss of ambulation, but also may have more side effects, although differences in dosing could account for these findings (2). A second recent study showed comparable benefit between deflazacort and prednisone, but greater weight gain with prednisone (12). The benefit of GC steroids on DMD muscle likely arises from multiple mechanisms and a balance between beneficial and detrimental effects.

One hallmark of muscle injury is disruption of the muscle plasma membrane. In DMD, the sarcolemma is weakened and more readily disrupted, thus representing a model of chronic muscle injury (13). Anxa6, which encodes annexin A6, was identified as a genetic modifier of muscular dystrophy in mice (14), and annexins A1 and A6 are directly implicated in sarcolemmal repair and resealing after muscle membrane injury $(15,16)$. Furthermore, annexins are known regulators of the immune system where they are thought 

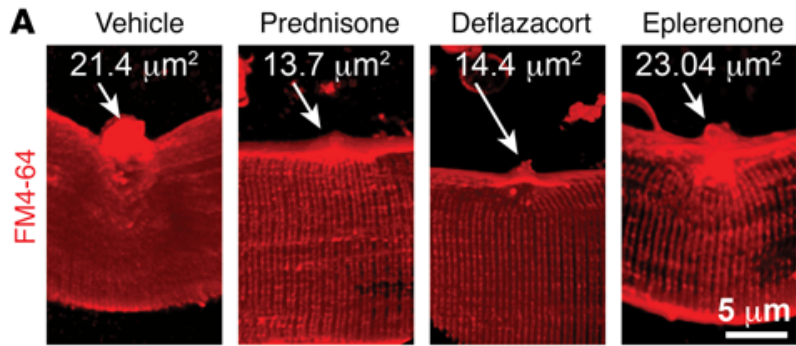

FM4-64
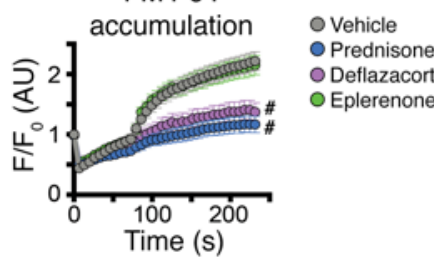

- Prednisone

O Deflazacort

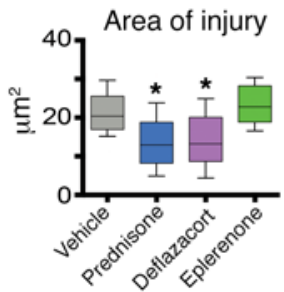

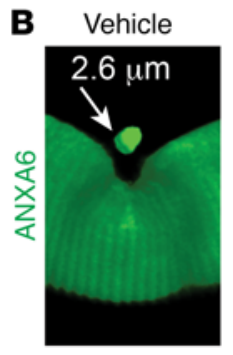
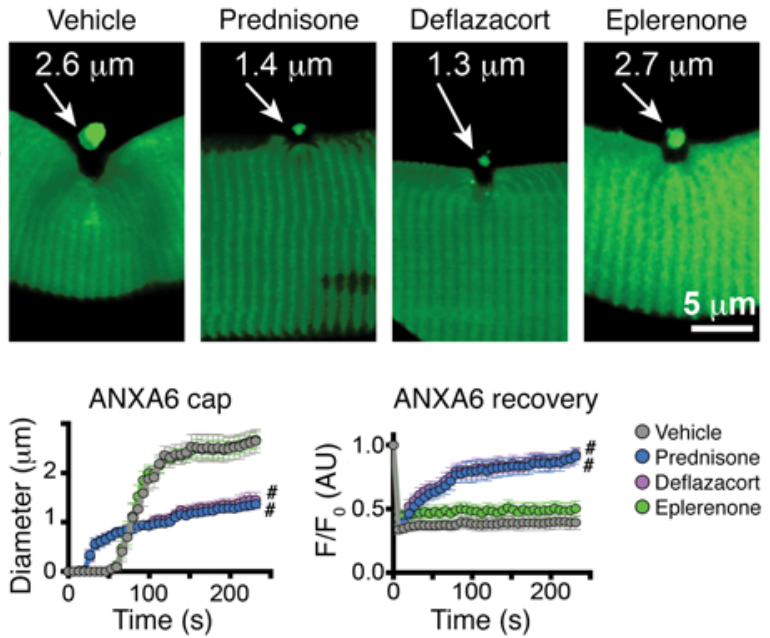

O Vehicle

- Prednisone

O Deflazacort

O Eplerenone

Figure 1. Pulse dosing of GC steroids improves sarcolemmal repair. Prednisone and deflazacort, both GC steroids, were given 1 day prior to injury. (A) Laser injury was applied to isolated muscle fibers in the presence of FM4-64, which marks sarcolemmal injury. A single dose of GC steroid reduced FM4-64 accumulation. Shown is Z-stack rendering of FM4-64 dye accumulation of laser-injured sarcolemmal sites at 300 seconds after injury. Quantitation shows that GC pulse associated with decreased dye accumulation over time as well as a decreased area of injury. (B) Imaging of annexin A6 (ANXA6) cap formation at the site of sarcolemmal injury. Pulse dosing of prednisone and deflazacort associated with smaller repair caps, consistent with reduced injury and enhanced repair. Shown is Z-stack rendering of GFP-tagged ANXA6 cap of laser-injured sarcolemmal sites at 300 seconds after injury. Quantitation of GC dosing demonstrated faster, smaller cap formation over time and faster recovery of GFP-tagged ANXA6 at injury site. FM4-64 and ANXA6-GFP pictures were acquired simultaneously. $F / F_{0}$, average fluorescence ratio versus average fluorescence at time 0 of imaging series. $n=50$ myofibers ( 5 mice)/group. ${ }^{*} P<0.05$ vs. vehicle, 1-way ANOVA test with Bonferroni's multiple comparison; ${ }^{\#} P<0.05$ vs. vehicle, 2 -way ANOVA test with Bonferroni's multiple comparison.

to promote resolution of the immune response (17). In leukocytes, Anxa1 is induced by GC steroids, suggesting that its upregulation is one mechanism by which GC steroids exert their effect (18).

Here, we compared the effect of prednisone and deflazacort on sarcolemmal repair in both normal and dystrophic myofibers. We found that a single pulse dose of either GC steroid enhanced sarcolemmal repair after laser injury. This effect was mediated by a direct upregulation of Anxa1 and Anxa6 through both direct transcriptional regulation and microRNA-regulated mRNA stability. We compared weekly versus daily GC steroid administration in murine models of acute muscle injury and chronic muscular dystrophy and found that both dosing schemes enhanced sarcolemmal repair. However, chronic daily dosing promoted atrophy with suppression of Klf15 and elevated F-box protein 32 (Fbxo32, encoding atrogin-1), and this atrophy program dominated any benefit from enhanced repair. These results point to dosing modulation to harness the beneficial effects of GC steroids on sarcolemmal repair and muscle recovery while avoiding the deleterious consequences of muscle atrophy.

\section{Results}

A single pulse of GC steroids improves sarcolemmal repair after inju$r y$ in normal muscle. We visualized muscle membrane repair in real time using high-resolution microscopy $(16,19)$. In this assay, a laser was used to disrupt the myofiber sarcolemma. Using this microwounding assay, it was previously shown that annexins assemble into a repair cap forming within seconds specifically at the site of laser ablation. FM4-64, a phosphatidylserine-binding dye, is used to mark the region of muscle injury. The flexor digitorum brevis (FDB) muscle of 8-week-old 129T2/SvEmsJ WT mice was electroporated with a plasmid to express GFP-tagged annexin A6 (ANXA6). Electroporation was conducted 7 days prior to the injury protocol in order to allow time for plasmid expression. Mice were treated with a single dose of steroids, either prednisone or deflazacort ( $1 \mu \mathrm{g} / \mathrm{g}$ body weight; ref. 20$)$, one day prior to harvesting myofibers for laser injury and live-cell imaging. Control mice were treated with vehicle or eplerenone $(100 \mu \mathrm{g} / \mathrm{g}$ body weight), an antimineralocorticoid steroid, as an additional pharmacological control.

FM4-64 dye accumulation, which measures the extent of membrane injury and resealing, was significantly lower in both prednisone- and deflazacort-treated myofibers compared with either eplerenone-treated or vehicle-treated myofibers (Figure 1A). The area of FM4-64 fluorescence was significantly reduced in both prednisone- and deflazacort-treated mice, as compared with vehicle- or eplerenone-treated myofibers (Figure 1A). This smaller injury area correlated with more efficient annexin repair cap formation. The annexin A6 cap appeared sooner and was smaller in steroid-treated myofibers compared with vehicle- or eplerenone-treated myofibers (Figure 1B). Moreover, annexin A6 recovery after photobleaching was significantly faster in steroidtreated myofibers (Figure 1B). Together, these findings demonstrate that a single pulse of GC steroids prior to injury accelerates and improves injury repair.

To verify the effectiveness of in vivo steroid dosing, splenocytes from treated animals were isolated and analyzed for markers of immune cell activation. In prednisone- and deflazacort-treated mice, Gzmb (encoding granzyme B) and Ifng (encoding IFN- $\gamma$ ) expression levels were downregulated compared with those of control animals, consistent with the anticipated effect of steroids 
A

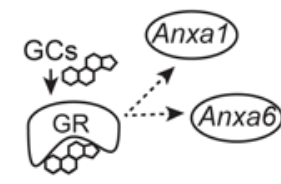

B



Whole muscle



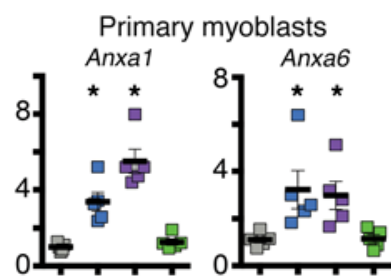

$\square$ Vehicle

Prednisone

Deflazacort

$\square$ Eplerenone
C

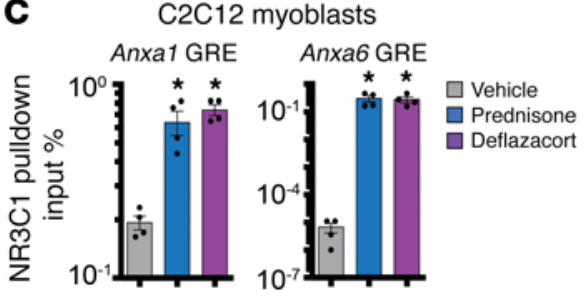

D

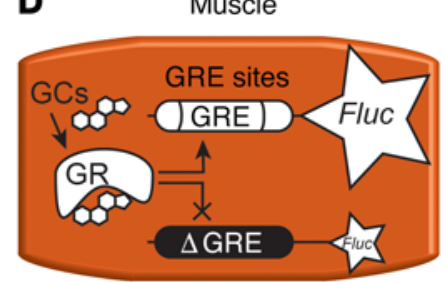

+ Prednisone

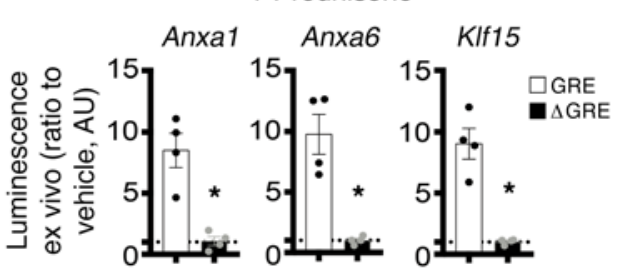

Figure 2. GC pulse upregulates Anxa1 and Anxa6 through increased GR binding of their GRE sites. (A) Model of transactivation pathway linking GC action in muscle to regulation of $A n x a 1$ and $A n x a 6$ expression; dashed lines, interrogated interactions. (B) Anxa1 and Anxa6 were upregulated in the skeletal muscle (quadriceps) and primary myoblasts (isolated from tibialis anterior) of mice 24 hours after a single pulse of GC steroids. (C) Twenty-four hours after a single GC pulse in C2C12 cells, ChIP-qPCR analysis showed that GRE sites of Anxa1 and Anxa6 were significantly enriched in CR occupancy as compared with vehicle. (D) Diagram shows experimental design of electroporation into muscles and 24 hours after i.p. prednisone pulse in WT mice. (E) Histograms show that prednisone pulse upregulated luciferase activity when constructs contained the GRE sites (white bars). Conversely, GC-related upregulation was ablated after deletion of the GRE-binding site in the constructs (black bars). KIf15 GRE was monitored as positive control. Data are expressed as fold change to luminescence from vehicle-treated muscles electroporated with the same plasmids (dashed line). $n=5$ mice/group (B); $n=4$ assays or mice/ group (C and $\mathbf{D}) .{ }^{*} P<0.05$ vs. vehicle, 1 -way ANOVA test with Bonferroni's multiple comparison $(\mathbf{B}$ and $\mathbf{C}) ;{ }^{*} P<0.05$ vs. WT site construct, unpaired 2-tailed $t$ test with Welch's correction (D).

(Supplemental Figure 1A; supplemental material available online with this article; https://doi.org/10.1172/JCI91445DS1).

Increased annexin gene expression through the GR. We next asked whether steroid treatment influenced expression levels of repair cap proteins in treated muscles. Anxal, encoding annexin A1, was previously reported as a downstream target of GC steroids in respiratory epithelial cells (21). We analyzed whole muscles as well as the myoblast fraction of muscle for annexin gene expression after steroid dosing. Primary myoblasts were isolated by sorting for CD56 (22). Both Anxa1 and Anxa6 mRNAs were significantly upregulated in both whole muscle and primary myoblasts after a single pulse of prednisone or deflazacort when compared with vehicle or eplerenone controls (Figure 2, A and B).

GCs act through GC receptor (GR, also known as NR3C1). We hypothesized that activated GR would drive Anxa1 and Anxa6 expression in muscle (Figure 2A). We identified GR-responsive elements (GRE; GnACAnnnTGTnC; ref. 23) upstream of the Anxa1 transcriptional start site at $-7610 \mathrm{bp}$ and upstream of Anxa6 at $-6639 \mathrm{bp}$. We interrogated these predicted binding sites for occupancy by GR in C2C12 myoblasts, cultured for 24 hours in the presence of either vehicle, prednisone, or deflazacort $(25 \mu \mathrm{g} /$ $\mathrm{ml}$ ). Chromatin immunoprecipitation (ChIP) using antibodies to GR and quantitative PCR (qPCR) was performed on the predicted GRE sites within Anxa1 and Anxa6 genomic loci. Occupancy of GRE sites by GR was significantly increased after a single dose of prednisone or deflazacort, as compared with vehicle (Figure 2C).

To confirm the role of GCs directly inducing annexin expression in skeletal muscle, we ligated promoter regions of Anxa1 and Anxa6 containing GREs upstream of a luciferase reporter plasmid. We generated both normal reporter constructs and those in which the GRE sequences were ablated ( $\triangle$ GRE). Plasmids were then electroporated into muscles of normal mice. One week following electroporation, animals were treated with a single dose of prednisone and muscles were collected for luciferase assays. Data were normalized to luciferase activity measured from muscles of vehicle-injected animals. The GRE upstream of Klf15 was used as positive control of GC responsiveness in muscle (11). A prednisone pulse induced increased luciferase activity from WT constructs as well as the Klf15 construct (Figure 2D). Conversely, the prednisone pulse failed to elicit a luminescence signal when the GRE sequences had been ablated (Figure 2D). These data are highly consistent with the studies conducted in $\mathrm{C} 2 \mathrm{C} 12$ cells and document that GCs promote the transcription of Anxa1 and Anxa6 in muscle.

In addition to direct transcriptional activation, GR-dependent transactivation of Anxa1 and Anxa6 was further reinforced through indirect activation of forkhead box O1 (Foxo1), which encodes a transcriptional activator that regulates muscle growth and atrophy (Supplemental Figure 1B) (24). The single pulse of GC steroids, but not of eplerenone, increased Foxo1 expression in muscle and primary myoblasts as compared with vehicle-treated animals (Supplemental Figure 1C). We found the same results when primary myoblasts were exposed to GC steroids in culture. We isolated primary myoblasts from WT mice that were age- and background-matched with mice used in previous analyses and cultured them for 24 hours (passage 0 ) in the presence of either vehicle, prednisone, or deflazacort $(25 \mu \mathrm{g} / \mathrm{ml})$. Expression of Anxa1, Anxa6, and Foxo1 was significantly upregulated in cells exposed to GCs as compared with those treated with vehicle (Supplemental Figure 1D).

Furthermore, a GRE site was identified within the first Foxo1 intron (+55284), and FOXO1-binding sites (GTAAACA; ref. 25) were 
A

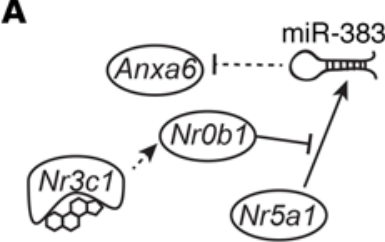

B


miR-383-

mimic
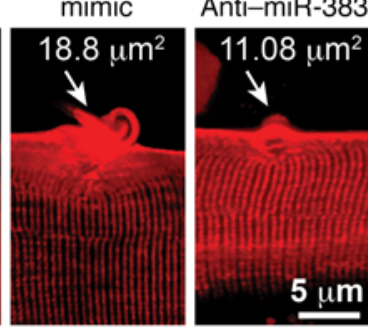

Normalized luminescence


$\mathbf{E}$

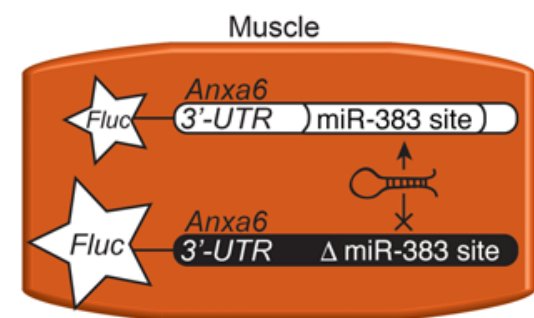



Time (s)



Figure 3. miR-383 contributes to GC-associated Anxa6 regulation. (A) Model of pathway linking GC uptake in muscle to regulation of miR-383 and Anxa6 expression. Solid lines, reported interactions; dashed lines, interrogated interactions. (B) NrOb1 and miR-383 were upregulated and downregulated, respectively, in GC-pulse dosed skeletal muscle (quadriceps) and primary myoblasts. (C) Electroporation directly into muscle with targeting oligonucleotides altered miR-383 and Anxa6 levels. (D) miR-383 mimic correlated with worse sarcolemmal injury, whereas anti-miR-383 correlated with smaller injury. (Left) Z-stack rendering of laser-injured sarcolemmal sites at 300 seconds after injury; (center) quantitation of FM4-64 dye accumulation at sarcolemmal site after laser injury; (right) end-point area of injury. (E) Luciferase assay to validate GC-dependent regulation of Anxa6-3' UTR targeting by miR-383 in skeletal muscle. Diagram depicting experimental design after introducing $3^{\prime}$ UTR reporter constructs into muscle followed by ex vivo Fluc measurement. miR-383 reduced luminescence, while 3' UTR without the miR-383-binding site ( $\Delta$ miR-383) increased luminescence. $n=5$ mice $/$ group (B); $n=3$ mice (30 myofibers)/group (C-E). ${ }^{*} P<0.05$ vs. vehicle, 1-way ANOVA test with Bonferroni's multiple comparison; ${ }^{\#}<0.05$ vs. vehicle, 2 -way ANOVA test with Bonferroni's multiple comparison.

found upstream of Anxa6 (-1872 bp), Foxo1 itself (-266 bp), and in the 5' UTR of Anxa1 (+628 bp). In GC-treated C2C12 myoblasts, GR binding to Foxo1 GRE appeared increased as compared with that in vehicle-treated cells (Supplemental Figure 1E). In C2C12 cells, GC steroids induced occupancy of FOXO sites by FOXO1 at the Anxa1, Anxa6, and Foxo1 loci (Supplemental Figure 1E). GC steroid activation of the Foxo1 GRE was also confirmed in intact muscle using luciferase assays on electroporated muscles and was dependent on the GRE as ablating the GRE sequence reduced reporter activity (Supplemental Figure 1F). GC steroids also induced activity of candidate FOXO sites in Anxa1, Anxa6, and Foxo1, and this also was dependent on the presence of FOXO sites (Supplemental Figure 1G). Thus, a single steroid pulse leads to the upregulation of Anxa1 and Anxa 6 in muscle through direct GR-driven transactivation and this response is reinforced through upregulation of Foxol, which in turn further drives annexin gene expression.

GC steroids sustain Anxa6 upregulation via miR-383 modulation. In silico prediction showed a putative binding site for the microRNA miR-383 in Anxa6 3' UTR (+228-247 bp from 3' UTR start). This binding site was hypothesized as an additional route by which GC steroids may contribute to Anxa6 upregulation. In this alternative pathway, nuclear receptor subfamily $\mathrm{O}$, group $\mathrm{B}$, member 1 (NrOb1), a reported target of the GR (NR3C1) in the adrenal cortex (26), would decrease miR-383 levels, thereby reducing miR-383-driven targeting of Anxa6 mRNA (Figure 3A). Consistent with this pathway, $\mathrm{NrOb1}$ was significantly upregulated and miR-383 significantly downregulated in muscle and in primary myoblasts after prednisone or deflazacort pulse, but not control treatments (Figure 3B).

To further validate miR-383 interaction with Anxa6's 3' UTR in muscle, we electroporated WT muscles with synthetic miR-383mimic and anti-miR-383 and interrogated the muscles for gene expression and sarcolemmal repair. Experiments were controlled with scramble miR-mimic and anti-miR. Expression levels of miR383 were significantly higher after miR-mimic and lower after anti-miR electroporation (Figure 3C). Anxa6 mRNA was reduced with miR-mimic and increased after anti-miR (Figure 3C). Sarcolemmal injury, area of injury, and rate of FM4-64 accumulation were significantly greater after miR-mimic and significantly smaller after anti-miR electroporation (Figure 3D). 


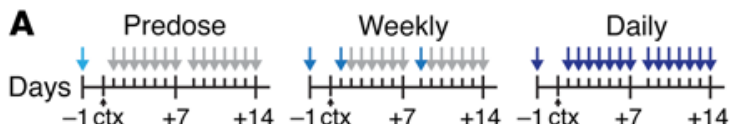

B

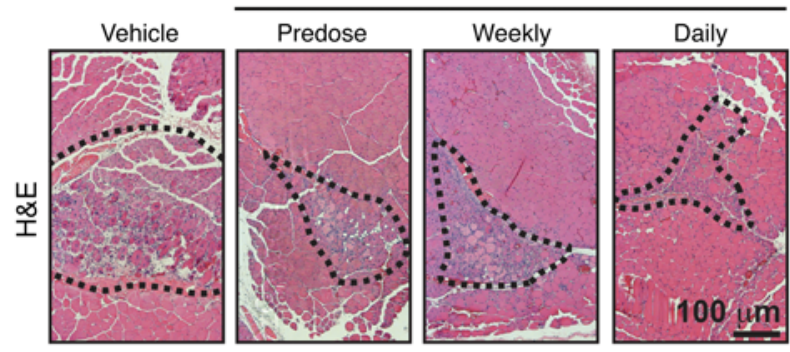

Prednisone

D



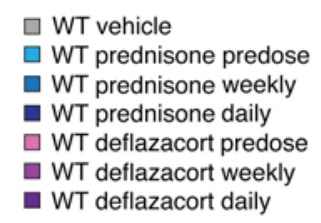



C

Infiltrating macrophages



No. of F4-80+ cells $/ \mathrm{mm}^{2}$

E

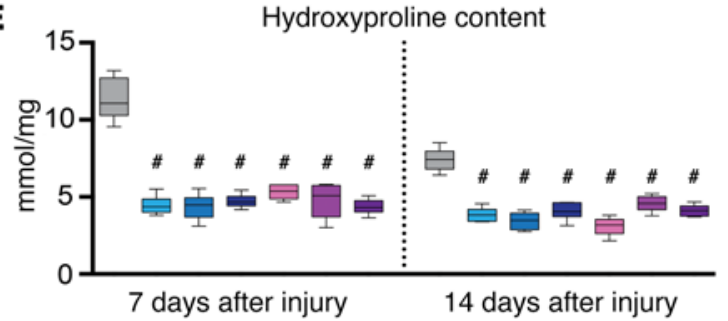

Figure 4. GC steroids decrease extent of acute muscle injury in WT muscle. Acute muscle injury was induced with cardiotoxin (ctx) injection in the tibialis anterior muscles of normal mice. (A) Diagram depicting the treatments performed in parallel in WT mice. Colored arrows, GC steroid injection; gray arrows, vehicle injections. (B) GC steroid regimens comparably reduced the extent of injury 7 days after cardiotoxin injection. (Left) Representative H\&E images of tibialis anterior muscles with prednisone treatments. The dotted lines outline the injury area, which includes necrosis, fibrosis, immune cell infiltrates, and centrally nucleated fibers. (Right) Injury extent quantitation (10 replicates). (C) GC regimens comparably decreased macrophage infiltration within the area of injury 7 days after cardiotoxin injection. Data are depicted as quantitation of $\mathrm{F} 4-80^{+}$cells/mm ${ }^{2}$ in gastrocnemius muscles by immunostaining. (D) GC steroid regimens comparably reduced serum CK at 24 hours after injury, with no significant changes after 7 days. (E) Fibrosis was comparably reduced in the presence of all GC regimens both 7 and 14 days after muscle injury. Data are depicted as quantitation of hydroxyproline content in gastrocnemius muscles. Gray, WT vehicle; light blue, WT prednisone predose; blue, WT prednisone weekly; dark blue, WT prednisone daily; light purple, WT deflazacort predose; purple, WT deflazacort weekly; dark purple, WT deflazacort daily. $n=6$ mice/group. ${ }^{*} P<0.05$ vs. vehicle, 1-way ANOVA test with Bonferroni's multiple comparison; ${ }^{\#} P<0.05$ vs. vehicle, 2-way ANOVA test with Bonferroni's multiple comparison.

To confirm responsiveness of Anxa6 3' UTR to miR-383 and GCs, we then tested whether mutation of the miR-383 site in Anxa6 3' UTR would remove steroid-dependent modulation. The Anxa6 3' UTR site with the intact or ablated miR-383 site ( $\Delta$ miR383) was placed downstream of a luciferase (Fluc) reporter gene. We then electroporated the constructs into muscles and evaluated the luminescence levels 24 hours after prednisone pulse. In the presence of the miR-383 site, the prednisone pulse was associated with increased luminescence (Figure 3E). Conversely, ablation of the binding site ( $\Delta$ miR-383 constructs) induced significantly higher luminescence levels, resulting in no difference between vehicle and prednisone treatments (Figure 3E). Thus, in addition to direct effects of steroids on Anxa6 gene expression, the GC steroid pulse reinforces Anxa6 upregulation by reducing miR-383-dependent targeting of Anxa6 3' UTR.

GC steroids improve recovery from muscle injury. The studies described above demonstrate that GC steroids enhance recovery from laser-induced disruption of the sarcolemma by upregulating annexins. We next assessed whether GC steroids improved recovery from acute focal in vivo injury by injuring muscles using an intramuscular injection of cardiotoxin (27). WT mice were given prednisone or deflazacort i.p. at $1 \mu \mathrm{g} / \mathrm{g}$ body weight 1 day before injury and then given daily or weekly steroids for 14 days (Figure
4A). Injury area and macrophage infiltration were comparably reduced with all steroid regimens (Figure 4, B and C, and Supplemental Figure 2A). Serum creatine kinase (CK) was elevated at 24 hours after injury and was significantly reduced by all steroid treatments (Figure 4D). By 7 days after injury, serum CK was no longer elevated. Injury-associated fibrosis, measured by hydroxyproline content and Masson's trichrome staining, was variably reduced by all steroid regimens at both 7 and 14 days after injury (Figure 4E). All steroid treatments, including the single predose, reduced inflammation and fibrosis after acute injury. These data indicate that a pulse of GC steroids improves recovery from muscle injury in vivo.

Daily GC steroid dosing drives atrophy. Chronic steroid treatment has been associated with muscle atrophy (28). To understand how differential GC dosing altered muscle repair, we compared weekly steroid administration versus daily administration in WT mice after acute injury. We assessed how steroid dosing affected muscle performance and molecular profile. A single weekly steroid dose increased running distance to exhaustion, while daily steroid dosing reduced exercise capacity compared with that of vehicle controls (Figure 5A). Ex vivo muscle performance was monitored in the tibialis anterior muscles. Consistent with in vivo performance, tetanic force was increased in weekly 
A



D

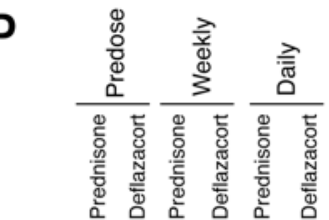

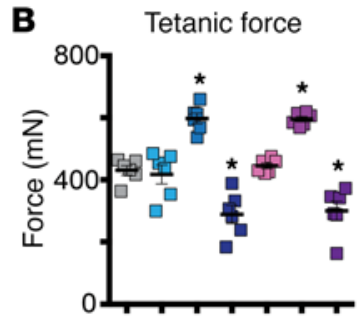

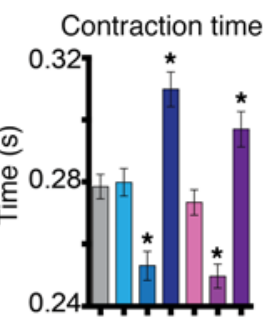

$\square$ WT vehicle

$\square$ WT prednisone predose - WT prednisone weekly

WT prednisone daily

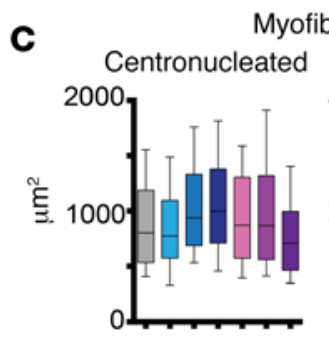

Myofiber CSA

$\square$ WT deflazacort predose

WT deflazacort weekly

- WT deflazacort daily
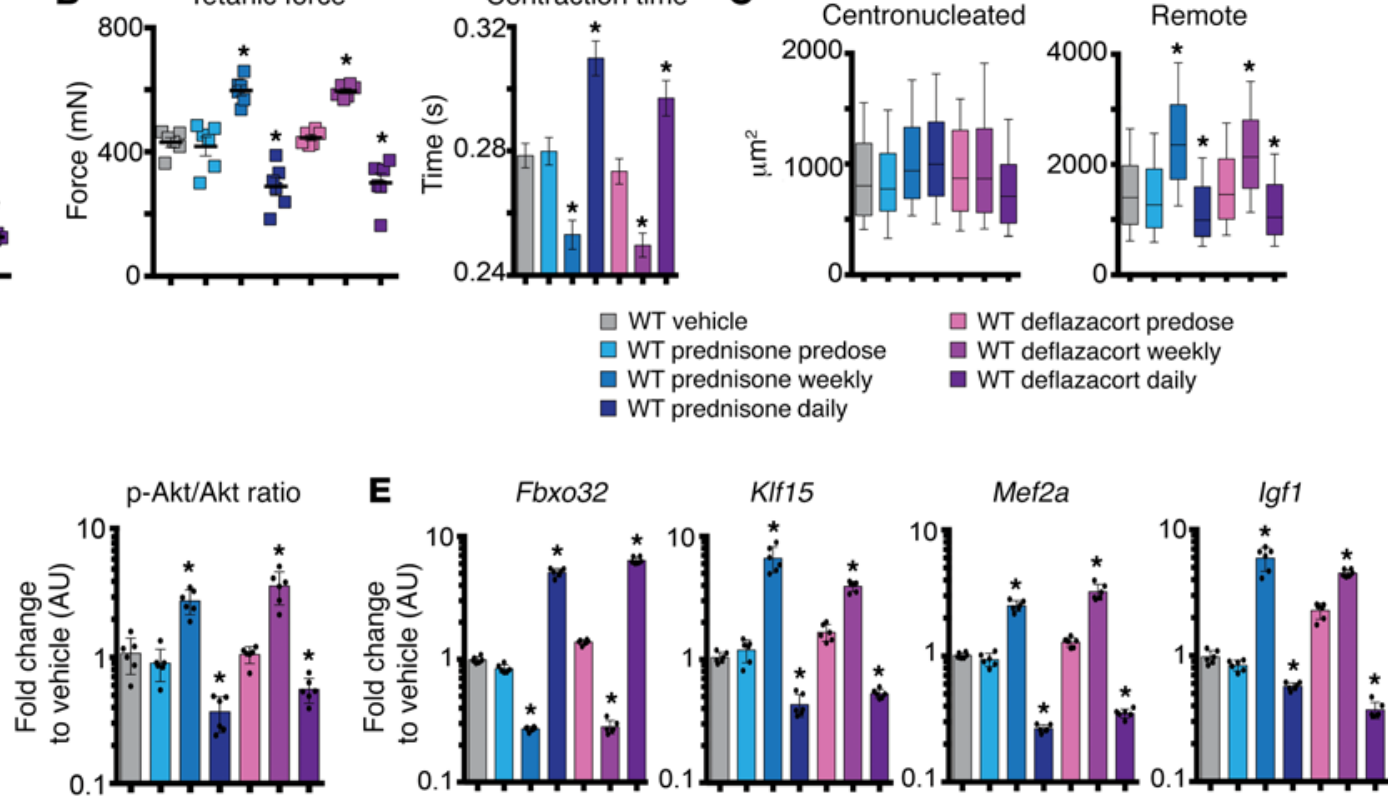

Figure 5. Weekly GC steroid dosing enhanced muscle performance, while daily GC treatments promoted atrophy in normal muscles 14 days after injury. (A) Weekly GC steroid dosing enhanced running distance after injury, while daily GC steroid dosing reduced performance. (B) Maximum tetanic force and contraction time were increased and accelerated, respectively, in tibialis anterior muscles of injured mice treated with weekly GC. Daily GC steroid dosing induced opposite effects. (C) CSA of myofibers remote from injury site was increased by weekly but not daily GC steroid dosing, while no significant change in CSA was seen within area of injury. (D) Representative blots and densitometric quantitation of p-Akt (Ser473) from duplicate blots run in parallel from tibialis anterior muscles 14 days after injury (3 replicates). (E) Fourteen days after injury, Fbxo32 gene expression was increased after daily GC dosing and reduced by weekly GC dosing. KIf15 was stimulated by weekly dosing and reduced after daily GC dosing. Daily GC steroid treatments induced opposite trends. Mef2a and Igf1 followed similar divergent trends. Gray, WT vehicle; light blue, WT prednisone predose; blue, WT prednisone weekly; dark blue, WT prednisone daily; light purple, WT deflazacort predose; purple, WT deflazacort weekly; dark purple, WT deflazacort daily. $n=6$ mice/group. ${ }^{*} P<0.05$ vs. vehicle, 1-way ANOVA test with Bonferroni's multiple comparison.

dosed and decreased in daily dosed mice (Figure 5B). Contraction time to maximum force was reduced after weekly steroid dosing and, correspondingly, delayed in muscles from mice that received daily GC administration (Figure 5B). These outcomes were dependent on repetitive dosing of GCs, since the predose group did not significantly differ from vehicle controls (Figure 5 , A and B). We evaluated the myofiber cross-sectional area (CSA) in injured muscles. We separately analyzed the CSA of myofibers within the injury area, as marked by myofibers with centrally placed nuclei, and those fibers remote from injury area (Supplemental Figure 2C). While the CSA of centronucleated myofibers did not significantly differ among groups, the CSA of remote myofibers was increased after weekly dosing and decreased after daily steroid administration (Figure 5C). To evaluate systemic effects on glucose metabolism, serum glucose from peripheral blood was measured and did not change among groups at 14 days after injury (Supplemental Figure 3A). These data demonstrate that weekly steroid dosing enhanced muscle recovery from injury. In contrast, daily steroid dosing impaired muscle performance, indicating that enhanced sarcolemmal repair was insufficient to overcome atrophy driven by daily CG steroid dosing.

To better understand the differential effects of steroid-dosing regimens, we examined gene expression and phosphorylated AKT levels in injured muscles 14 days after cardiotoxin injection. Both weekly and daily steroid treatments were associated with increased levels of Anxa1 and Anxa6 expression, accounting for the reduced injury response (Supplemental Figure 3B). Phosphorylated AKT (Ser473; p-AKT) is a marker of hypertrophic muscle remodeling and is reduced when muscle mass is lost (29). p-AKT levels were significantly increased after weekly regimens and significantly reduced after daily GC dosing (Figure 5D). We analyzed Fbxo32, which encodes atrogin-1 (also known as MAFbx), an important mediator of muscle atrophy $(30,31)$. We also monitored Klf15, a transcription factor that interacts with GR pathways and mediates steroid-dependent ergogenic effects in skeletal muscle $(11,32)$. Fbxo32 was upregulated in muscle of daily dosed mice, paralleling atrophic remodeling, and suppressed by weekly dosing. Klf15 was suppressed by daily dosing and upregulated in weekly dosed mice (Figure 5E). Consistent with the expression pattern of Klf15, genes within its downstream metabolic pathway were upregulated by weekly administration and downregulated by daily dosing 14 days after injury (Supplemental Figure 3C).

To confirm that GC-associated molecular changes derived from myofibers, we isolated the myofiber-enriched fraction from GC- and vehicle-treated injured muscles 14 days after injury (Supplemental Figure 3D). qPCR of the myofiber fraction recapitulated the findings observed in whole muscle. Namely, these data showed that both daily and weekly steroid dosing was sufficient to upregulate repair pathways, but that daily dosing induced Fbxo32 and repressed Klf15 (Supplemental Figure 3E). Therefore, although daily GC steroid dosing improved repair after acute injury, this beneficial effect was negated by profound atrophic remodeling. 
A
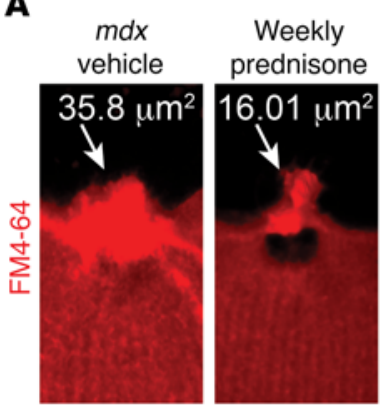

Daily
rednisone
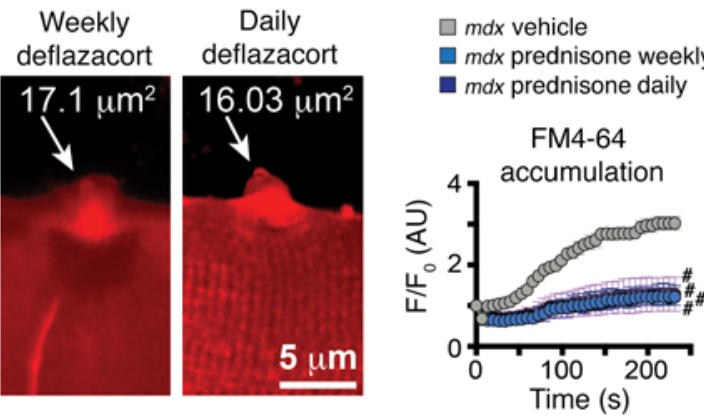

$m d x$ deflazacort weekly
$m d x$ deflazacort daily
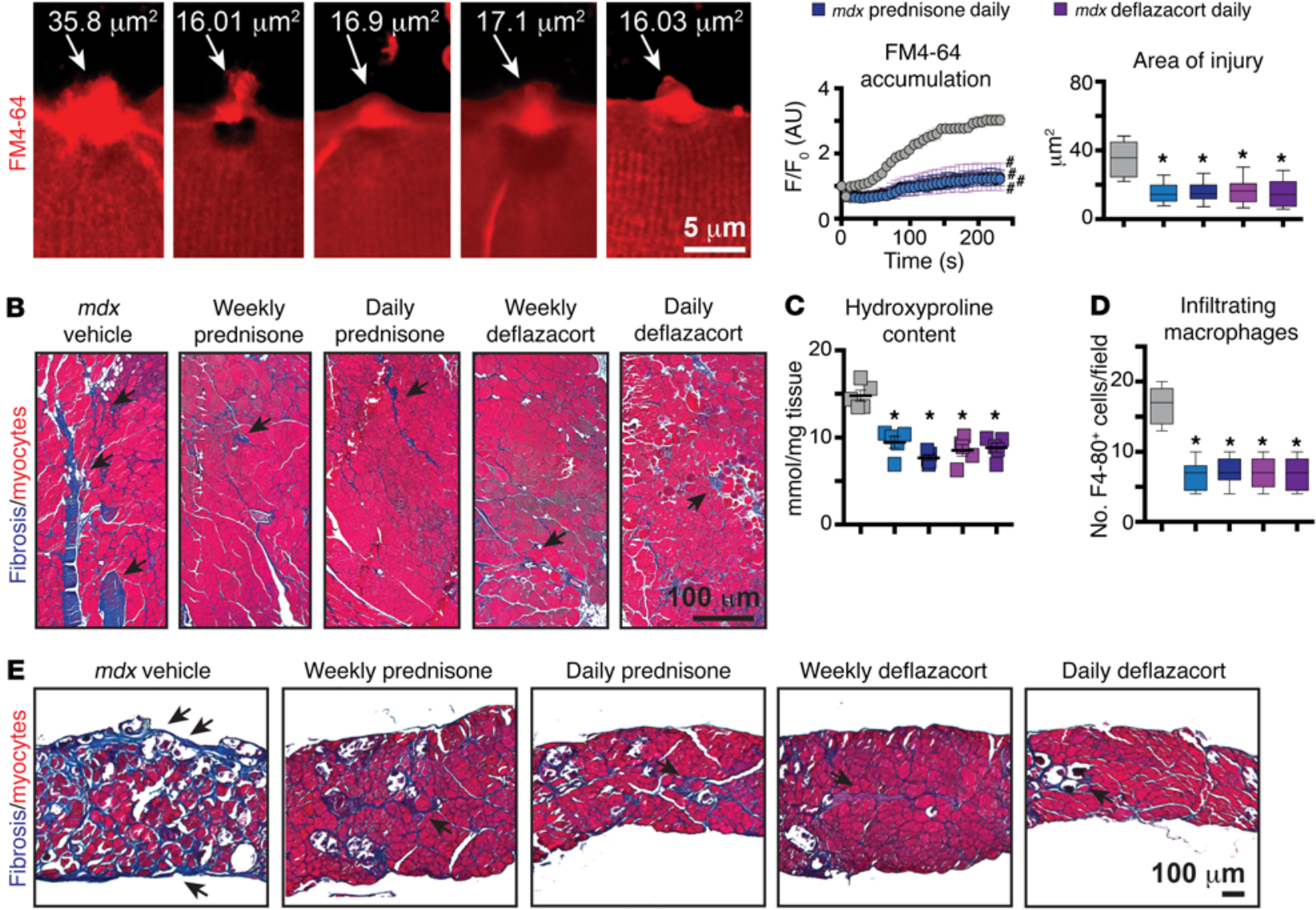

Figure 6. Weekly and daily GC steroid regimens enhanced sarcolemmal repair in $\mathbf{m d x}$ mice. Steroids were given to $m d x$ mice daily or weekly for 4 weeks. (A) (Left) Weekly and daily GC steroid regimens reduced FM4-64 dye accumulation and sarcolemmal injury in myofibers. Z-stack rendering of FM4-64 dye accumulation at laser-injured sarcolemmal sites at 300 seconds after injury. (Right) Quantitation of FM4-64 dye accumulation at sarcolemmal site after laser injury and end-point area of injury. (B) Fibrotic infiltration (blue scars; arrows) was reduced after all GC regimens, as evidenced by Masson's trichrome staining of gastrocnemius muscle sections (10 replicates). (C) GC steroid regimens reduced hydroxyproline content in quadriceps muscles. (D) Macrophage infiltrates in skeletal muscles were reduced after all GC regimens. Chart shows quantitation of infiltrating F4-80+ cells, assessed by immunostaining on quadriceps muscle sections. (E) Diaphragm muscles followed trends similar to those of hind limb muscles, as fibrosis (blue scars; arrows) appeared comparably reduced after all GC regimens (10 replicates). $n=5$ mice (50 myofibers)/group. ${ }^{*} P<0.05$ vs. vehicle, 1-way ANOVA test with Bonferroni's multiple comparison; ${ }^{P} P<0.05$ vs. vehicle, 2 -way ANOVA test with Bonferroni's multiple comparison.

Weekly and daily steroid treatments promote sarcolemmal repair in $m d x$ dystrophic mice. Chronic daily steroid administration is associated with adverse effects including obesity and, in children, growth suppression (33). Studies of prednisone administration in children with DMD have suggested that weekend dosing has comparable beneficial effects compared with daily dosing (34). In the $m d x$ model, weekly dosing was associated with a sustained long-term benefit compared with that in control-treated mice (35). However, there has been limited widespread uptake of weekly steroid regimens, so we investigated the effects of weekly and daily steroid dosing in an animal model of muscular dystrophy. These studies were conduced in 6-month-old $m d x$ male mice on a $D B A / 2 J$ background, since these mice bear a closer resemblance to human DMD pathology and specifically the age at which steroid dosing is often initiated (36). Mice were given prednisone and deflazacort i.p. at $1 \mu \mathrm{g} / \mathrm{g}$ body weight either weekly or daily, and results were analyzed after 4 weeks of treatment. Sarcolemmal injury and repair of isolated dystrophic myofibers were improved comparably by both steroid-dosing treatments, with both groups showing reduced FM4-64 dye accumulation and area of injury when compared with vehicle (Figure 6A). There were no differences between prednisone and deflazacort, indicating that the types of GC steroid and dosing regimen were comparable in their effect on response to laser-induced sarcolemmal injury.

We correlated steroid-induced repair enhancement with serum CK levels, fibrosis, and immune infiltration within dystrophic skeletal muscle. Serum CK was comparably reduced by all steroid regimens (Supplemental Figure 4A). Fibrotic infiltrates were comparably reduced in gastrocnemius muscles among steroid groups, as compared with vehicle controls (Figure 6B). Quantitation of hydroxyproline levels in quadriceps muscles revealed a significant reduction of hydroxyproline content in all steroidtreated mice compared with vehicle-treated controls (Figure 6C). Inflammation is a prominent feature in many forms of muscular 
A
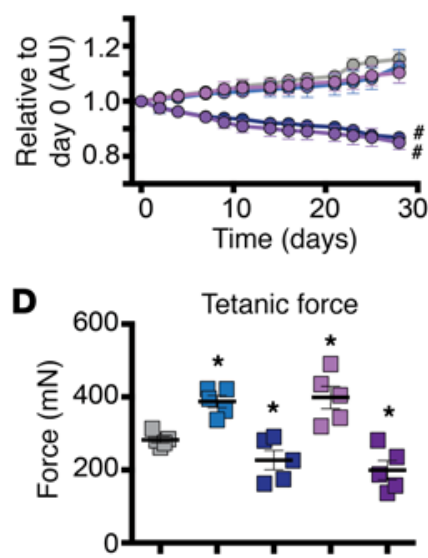

B

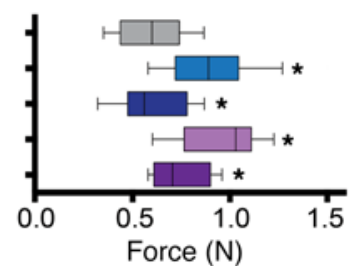

E Fatigue analysis



Isometric contraction

bouts

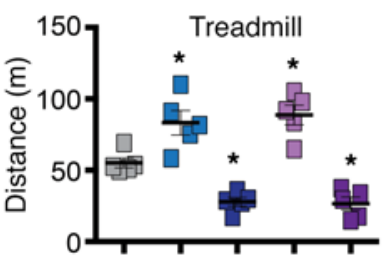

C Myofiber CSA

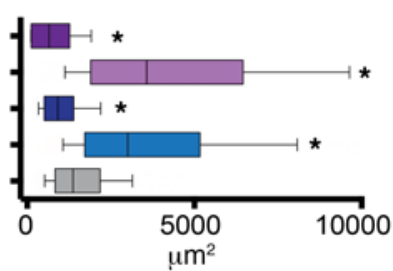

F Plethysmography
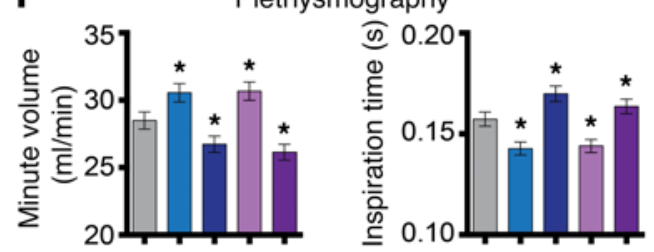

G

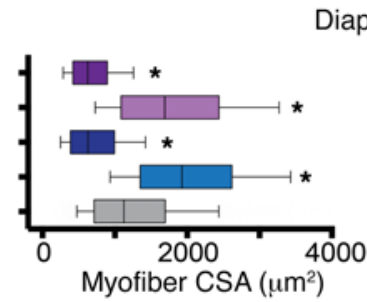

Diaphragm

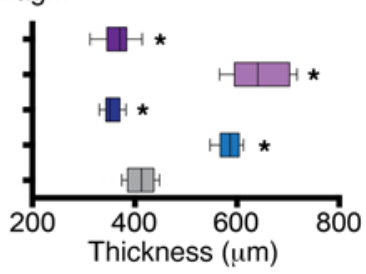

$\square m d x$ vehicle

$\square m d x$ prednisone weekly

mdx prednisone daily

$m d x$ deflazacort weekly

- $m d x$ deflazacort daily

Figure 7. Daily GC dosing elicits atrophic $\mathbf{m d x}$ skeletal muscles, while weekly GC dosing does not. (A) Daily, but not weekly, GC steroid dosing caused loss of body mass. (B) Weekly GC steroid dosing enhanced grip strength and run-to-exhaustion performance, while daily GC steroid-dosing treatments correlated with reduced grip strength and run performance. (C) Myofiber CSA was increased after weekly, but decreased after daily GC administration in gastrocnemius muscle sections. (D) Max tetanic force of tibialis anterior muscles was increased after weekly treatments and decreased after daily GC administration. (E) Fatigue analysis showed that tetanic force was increased over consecutive contraction bouts after weekly dosing, while daily dosing induced opposite trends. (F) Weekly GC regimen correlated with improved respiratory function, as assessed by WBP. Minute volume was increased and inspiration time was decreased in weekly GC dosing. Daily dosing reversed these beneficial trends. (C) Weekly GC treatments promoted increased CSA and diaphragm thickness, while daily CG dosing reduced CSA and diaphragm thickness. $n=5$ mice/group. ${ }^{*} P<0.05$ vs. mdx vehicle, 1 -way ANOVA test with Bonferroni's multiple comparison; ${ }^{\#} P<0.05$ vs. $m d x$ vehicle, 2-way ANOVA test with Bonferroni's multiple comparison.

dystrophy, including DMD (37). Macrophage infiltration was significantly reduced in quadriceps muscles of all steroid-treated mice compared with controls (Figure 6D). Fibrosis was reduced by all GC regimens in the diaphragm muscle (Figure 6E), which was analogous to what occurred in hind limb muscles. Thus, both weekly and daily steroid treatments enhanced sarcolemmal repair and reduced fibrosis and immune cell infiltration to a comparable extent in dystrophic muscles.

Daily steroid dosing triggers atrophy, while weekly dosing ameliorates muscular dystrophy features in mdx mice. To monitor for muscle atrophy, body weight, muscle performance, and histopathology were assessed in steroid-treated $m d x$ mice. Body weight significantly declined during daily steroid treatment, while $m d x$ mice receiving weekly steroids did not lose body mass compared with vehicle controls (Figure 7A). Grip strength significantly increased after weekly steroid treatments compared with vehicle controls, while daily dosing resulted in decreased grip strength compared with controls (Figure 7B), although, when results were normalized to body weight, no differences were found among groups (Supplemental Table 1). To further examine muscle performance, mice were subjected to incline treadmill run test until exhaustion. Weekly steroid-injected $m d x$ mice ran further than controls, while daily dosed mice ran less than controls, indicating that weekly and daily treatments had opposing effects on muscle performance (Figure 7B).

These functional changes were reflected in muscle size and fiber type. Myofiber CSA analysis of the gastrocnemius muscle revealed a significant increase in myofiber size after weekly steroid treatments compared with vehicle-treated control. There was a corresponding decrease in myofiber CSA after daily steroid dosing compared with vehicle-treated muscles (Figure 7C). Myofiber typing of quadriceps muscle revealed that weekly steroid treatment was associated with an expansion of fast glycolytic type $2 \mathrm{~B}$ fibers and a decrease in the number of fast oxidative type $2 \mathrm{~A}$, while daily steroid treatments correlated with the opposite trend compared with vehicle (Supplemental Figure 4, B and C). Moreover, daily steroids induced a shift of type 2A myofiber CSA toward lower values compared with weekly dosed and vehicle treated. Conversely, weekly dosing shifted toward larger type $2 \mathrm{~B}$ myofibers. However, these trends were not significant when fiber type CSA was compared across treatments for both variables (Supplemental Figure 4D).

Force, fatigue, and contraction/relaxation time analyses were analyzed in tibialis anterior muscles in live, anesthetized mice. Tetanic force increased after weekly steroid treatment compared 
A
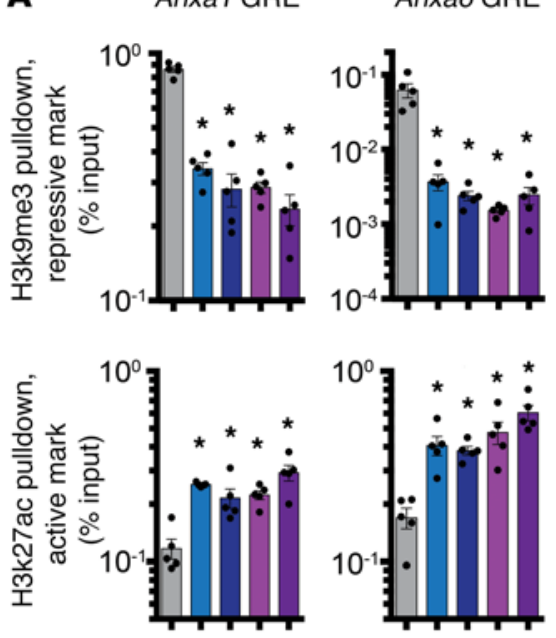

B

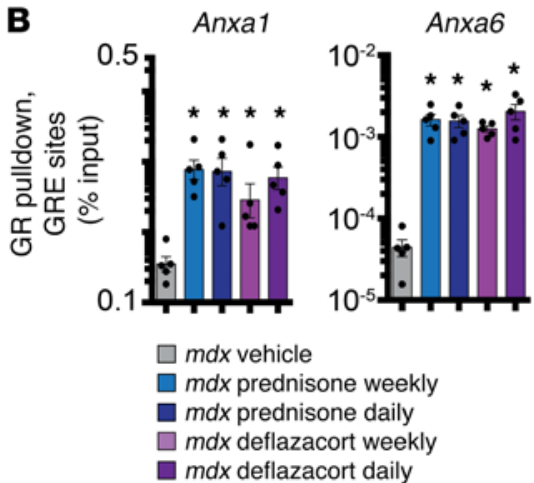

with that in control-treated $m d x$ mice (Figure 7D), although specific force was unchanged, indicating that this increase in force reflected the increase of muscle mass (Supplemental Figure 4E). Conversely, daily steroid treatment reduced tetanic force compared with that in vehicle-treated controls, and this also reflected the decline in muscle mass, as specific force was not changed. Fatigue analysis over 25 repeated isometric contractions showed that curves of tetanic force were consistently higher in weekly treated and lower in daily treated muscles as compared with vehicle treated (Figure 7E). Also, times of contraction and relaxation to/from maximum tetanic force were reduced after weekly dosing, but increased after daily regimens (Supplemental Figure 4E).

Taken together, these data show that weekly steroid treatments were associated with increased muscle mass and a proportional increase in strength, with lower relative resistance to fatigue and faster contraction/relaxation. Conversely, daily steroid treatment triggered atrophic remodeling. These data indicate that dystrophic muscle, like normal muscle, benefits from weekly dosing through enhanced sarcolemmal repair and reduction of fibrosis and immune infiltrate, while escaping the atrophic pathways driven by daily steroid dosing.

GC steroid dosing improves cardiopulmonary function in $m d x$ mice. We next assessed whether the same trends were apparent for respiratory function and diaphragm muscle. Respiratory function was measured utilizing whole-body plethysmography (WBP) on resting $m d x$ mice at the end point of treatment. Respiratory minute volume increased in weekly GC-treated mice and decreased
Figure 8. Histone mark enrichment in GRE sites in gastrocnemius myofibers of treated $\boldsymbol{m d x}$ mice and GR occupancy. (A) ChIP-qPCR for the repressive histone mark pH3k9me3 and the active histone mark H3k27ac (active histone mark) revealed that GREs of Anxa1 and Anxa6 were comparably enriched in permissive histone signature (higher $\mathrm{H} 3 \mathrm{k} 27 \mathrm{ac}$, lower $\mathrm{H} 3 \mathrm{k} 9 \mathrm{me} 3$ ) after all GC treatments. However, the KIf15 GRE presented a divergent histone signature in response to weekly and daily regimens, consistent with its gene-expression trends. (B) After 4 weeks of GC treatment, ChIP-qPCR on the myofiber fraction from gastrocnemius muscles revealed that CRE sites were enriched in GR (NR3C1) binding in all steroid-treated muscles. GR occupancy on KIf15 was significantly higher after daily GC administration, as compared with weekly regimens, consistent with dose-dependent occupancy. $n=5$ mice/group. ${ }^{*} P<0.05$ vs. $m d x$ vehicle or indicated sample, 1-way ANOVA test with Bonferroni's multiple comparison.

in daily GC-treated mice as compared with those treated with vehicle control. Time of inspiration was decreased in weekly GCtreated mice and increased in daily GC-treated mice (Figure 7F). Myofiber CSA and transverse diaphragm muscle thickness were increased after weekly GC injection and decreased after daily GC administration (Figure 7G). Thus, similarly to what occurred in hind limb muscles, weekly GC treatment resulted in improved respiratory function and diaphragm pathology, while daily GC treatment correlated with atrophy, irrespective of reduced fibrosis.

We monitored heart fibrosis and blood pressure in $m d x$ mice to assess possible effects of GC steroid treatments. Histologic assessment of left and right ventricle walls showed that all GC treatments correlated with decreased fibrosis in both ventricle walls, paralleling the effects observed in muscle (Supplemental Figure 5A). Myocardial fibrosis associates with increased ventricle strain (38). Left ventricle strain was assessed by echocardiography. Consistent with the reduction in fibrosis, longitudinal strain and circumferential strain were reduced in all steroid-treated mice compared with vehicletreated mice, with no significant changes recorded between weekly and daily regimens (Supplemental Figure 5B). Systolic and diastolic blood pressure, although variable within each cohort, did not significantly change with treatment (Supplemental Figure 5C). Thus, fibrosis and strain were comparably reduced in dystrophic ventricles after 4 weeks of either weekly or daily GC treatments, with no significant effects on blood pressure.

Gene regulation is associated with regimen-specific remodeling of dystrophic muscle. We next interrogated dystrophic muscles of treated and control mice for expression levels of candidate genes involved in sarcolemmal repair and atrophy. We assessed the expression of specific genes from whole muscle tissue as well as the isolated myofiber fractions from vehicle and weekly and daily GC steroid regimens. As in WT muscle, Anxa1 and Anxa6 were upregulated by all GC regimens (Supplemental Figure 6A). Fbxo32 expression levels were significantly higher after daily steroid treatments and lower after weekly steroid treatments, while Klf15 levels were increased by weekly steroids and suppressed by daily steroid treatment (Supplemental Figure 6A).

To gain further insight into differential regulation of these genes, we examined chromatin marks and GR occupancy on the GREs of Anxa1, Anxa6, and Klf15. Studies were conducted at treatment end point using the myofiber fraction of vehicle and treated $m d x$ muscles. We monitored genomic elements for enrichment in the repressive histone mark histone-3 tri-methyl-lysine-9 
A

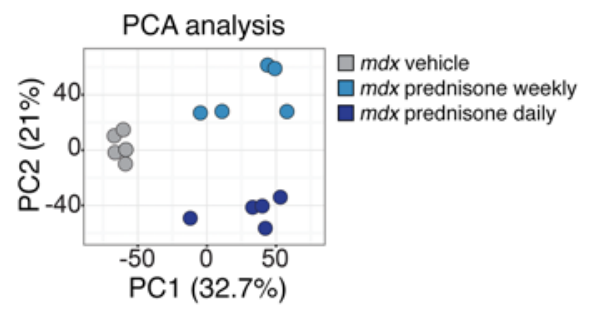

B



C Enrichment in gene ontology terms (biological process)


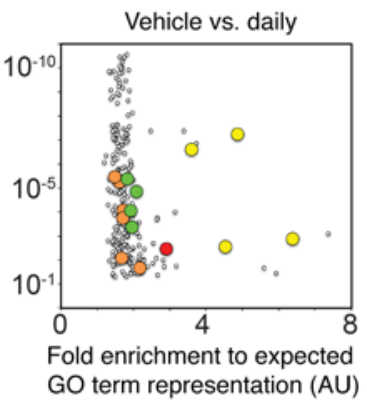

E



Fold change to row RPKM mean

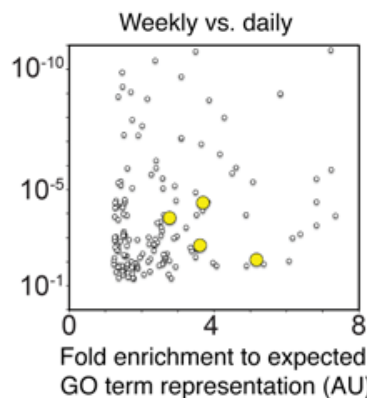

Membrane repair

Immune response

Steroid metabolism

$\sqrt{2}$ 
ased clustering of samples using principal component analysis segregated transcriptional profiles according to dosing regimen (Figure 9A and Supplemental Data Set 1). Unsupervised hierarchical clustering confirmed clustering based on dosing regimen (Figure 9B). The differential gene subsets from each binary comparison (vehicle versus weekly, vehicle versus daily, weekly versus daily) were analyzed for enrichment of gene ontology (GO) terms of interest. Among all enriched GO terms, GO terms related to membrane repair, tissue growth, immune response, and steroid metabolism were significantly enriched when comparing prednisone-treated muscles with vehicle-treated muscles (Figure 9C; Supplemental Data Set 2). When comparing weekly versus daily treated muscle to vehicle-treated muscle for the GO terms listed above, only the GO terms related to steroid metabolism were significantly enriched (Figure 9C; Supplemental Data Set 2). Thus, both weekly and daily prednisone regimens induced gene expression changes in membrane repair and tissue growth pathways in muscle. However, the metabolic response to steroids differed between weekly and daily GC administration.

Heat maps of gene-relative increases in sequencing reads (reads per kilobase of transcript per million reads [RPKM]) showed that sarcolemmal repair genes, including Anxa1 and Anxa6, were upregulated in both GC-treated muscles, as compared with vehicletreated muscles (Figure 9D; Supplemental Data Set 3). Genes associated with muscle atrophy were generally downregulated in weekly treated, but upregulated in daily treated muscles, including Fbxo32 (Figure 9E; Supplemental Data Set 3) (40). Conversely, genes associated with the KLF15 pathway were upregulated after weekly administration, but downregulated after daily dosing, as compared with vehicle-treated muscles (Figure 9F; Supplemental Data Set 3). Thus, the regimen-associated trends in sarcolemmal repair and muscle remodeling were recapitulated in the wholemuscle transcriptome analysis.

We compared profiles from weekly versus daily treated $m d x$ muscle. No significant changes in Anxa1 and Anxa6 transcript abundance were seen between weekly and daily dosing. Compared with weekly dosing, daily prednisone administration was associated with upregulation of Fbxo32 and Mstn, encoding the negative regulator of muscle mass myostatin (Supplemental Figure 7; Supplemental Data Set 4) (41). Conversely, weekly GC dosing was associated with upregulation of Klf15 and, to a lesser extent, Ifg1. Thus, weekly dosing activates key aspects of the beneficial effects of steroids in muscle and recovery from injury without triggering atrophy.

\section{Discussion}

GC steroids improve muscle repair after injury. Skeletal muscle myofibers are susceptible to plasma membrane disruption, likely due to elongated shape and contraction-induced stress (42). Here, we report direct effects of GC steroids on sarcolemmal repair. We evaluated both prednisone and deflazacort and found that both stimulated annexin gene expression equally. Although it has been suggested that the properties of deflazacort may be associated with fewer metabolic adverse effects (43), we found little difference between prednisone and deflazacort in their ability to improve recovery from injury. A single pulse of GC steroids correlated with upregulation of annexin genes in muscle, faster formation of the annexin repair cap, and faster recovery of annexins to the injury site. GC treatment, whether daily or weekly, reduced the extent of injury in both WT and dystrophic muscles. GC steroids improved sarcolemmal repair not only in muscular dystrophy, but also after acute muscle injury in normal muscle.

Notably, GC steroid treatment in dystrophic muscle upregulated many genes encoding proteins implicated in muscle repair (16); thus, the effect of GC steroids on recovery from injury is not just limited to annexin gene expression. Transcriptome analysis showed that genes including caveolin 3 (Cav3), myoferlin (Myof), dysferlin (Dysf), and Trim72 (encoding MG53) were all increased $(19,44,45)$. More refined studies are needed to shed light on whether transcriptional regulation of those genes is directly stimulated by GCs or is an indirect effect of enhanced muscle repair. It is also important to consider that other molecular pathways likely contribute to improving repair. For example, GC steroids are known to alter membrane lipid composition, and this altered composition may facilitate repair after injury (46).

Daily steroid dosing elicits atrophy, while weekly dosing does not. Weekly dosing of GC steroids improved muscle recovery from injury in both the acute setting of normal muscle and the chronic setting of muscular dystrophy. We did not observe significant differences in the type of CG steroid in that both prednisone and deflazacort yielded similar results in repair assays. A recently published phase III clinical trial found comparable effects on the muscle strength of boys with DMD treated with prednisone and deflazacort over 12 weeks, using dose ranges that were comparable to those used in our study, although prednisone was associated with more pronounced weight gain than deflazacort (12). Both weekly and daily steroid regimens induced a comparable reduction in fibrosis and ventricle strain in the heart of treated mice. Additional studies are needed, as GC steroid effects in DMD cardiomyopathy are debated, with contrasting reports of beneficial effects in DMD patients and detrimental effects in DMD models $(47,48)$. In skeletal muscle, molecular pathways of atrophy were triggered by daily dosing and this was accompanied by reduced performance of voluntary hind limb and respiratory skeletal muscles. In contrast, weekly dosing did not trigger atrophy and instead was associated with improved muscle performance.

Weekly GC treatment increased Klf15, while daily dosing suppressed Klf15. KLF15 is a known target of GR (32), and loss of Klf15 is known to exacerbate the skeletal muscle phenotype in $m d x$ mice (11). Muscle-specific upregulation of KLF15 was shown to improve muscle performance in $m d x$ mice, and it is known that KLF15 regulates not only glucose and amino acid use, but also lipid flux in muscle $(11,49)$. The striking divergent Klf15 transcriptional pattern elicited from weekly versus daily GC steroid dosing was reflected in a similar pattern of KLF15-regulated genes, seen in both candidate gene expression and in broad transcriptional profiling. KLF15 has also been implicated in diurnal regulation of gene expression in the heart (50), and so it is notable that all injections in this study were performed at $7 \mathrm{am}$, when mice have naturally low levels of cortisol (51). This pattern is the opposite of that in humans, where the highest peak of cortisol is seen in the early morning (52), and so in translating this finding to humans, time-of-day dosing may be relevant.

Ergogenic factors such as KLF15 improve muscle performance through a number of mechanisms, including substrate use (49). 
In this work, we extend this concept to gene-encoding proteins essential for repair. Thus, an important aspect of muscle performance includes the ability of muscle to recovery from injury, including exercise-induced injury. In addition to weekly GC dosing increasing Klf15, upregulation of Igf1 and Mef $2 a$ was also seen. Thus, efficient ergogenesis likely relies on fuel substrates, growth, and sarcolemmal repair processes.

Regimen-associated divergence in elicited muscle response. Klf15 is a direct target of the GR (32, 53-55). We found divergent epigenetic chromatin marks on the Klf15 GRE that paralleled the change in gene expression elicited by differential GC steroid dosing. Interestingly, GR (NR3C1) occupancy demonstrated a dose-dependent difference in occupancy of the Klf15 promoter, suggesting that the level of GR occupancy elicits differential effects. Muscle-specific deletion of the GR results in larger muscle mass and a reduction in adipose tissue, demonstrating crosstalk between these tissues (56). Furthermore, mTOR can counterbalance GR effects in muscle and thus may also represent a means of subverting aspects of the atrophy pathway triggered by daily steroid dosing (55).

In summary, our data indicate that intermittent weekly dosing of GC steroids better harnesses the dual beneficial effects on skeletal muscle, i.e., (a) improved sarcolemmal repair and (b) ergogenic remodeling. When chronic treatment is required, weekly dosing was associated with benefit without driving atrophic pathways. Furthermore, single pulse dosing of GC steroids in normal muscle improved repair by increasing repair pathways in muscle, indicating that repair pathways are relevant for the ergogenic process of both normal and diseased muscle.

\section{Methods}

Animals. The WT mice on a 129T2/SvEmsJ background were used for studies involving acute injury protocols. The $m d x$ mice on a DBA/2J background were obtained from The Jackson Laboratory (stock 013141) and interbred. Male mice were used for reported experiments. Mice were housed in a specific pathogen-free facility.

Plasmids. GFP-tagged annexins A1 and A6 were described previously $(14,16)$. The 3 ' UTR of Anxa6 was PCR cloned from cDNA from 129T2/SvEmsJ skeletal muscle and ligated into the pmirGlo plasmid (catalog 1330, Promega) using the SacI-XhoI sites; the miR-383-binding site (5'-CUGCAUUCAACUCUGAUC- ${ }^{\prime}$ ) was ablated by means of a Phusion Site-Directed Mutagenesis Kit (catalog F541, Thermo Scientific). Luciferase plasmids containing promoter fragments were obtained by cloning genomic sequences into pGL4.23 (E8411, Promega) using the KpnI-XhoI sites. Cloned genomic sequences from 129T2/SvEmsJ mice were used for GRE/FOXO site analysis (Supplemental Table 2).

Electroporation, myofiber isolation, and laser injury. FDB fibers were transfected by in vivo electroporation. Methods were as described previously (57) with modifications also described (58). For quantitative analysis of FM dye, fluorescence was measured at the site of injury in individual frames using Image $(\mathrm{NIH})$ and adjusted to baseline fluorescence at time 0 calculated at the membrane prior to damage (F/FO). This method allows comparisons of all strains and reduces variability introduced by differences in dye uptake or binding at time 0 . Analysis was conducted with the investigator blinded to treatment group.

Drug treatments. Prednisone, deflazacort, and eplerenone (catalog P6254, SML0123, E6657, Sigma-Aldrich) were resuspended in DMSO (catalog D2650, Sigma-Aldrich) at $5 \mathrm{mg} / \mathrm{ml}, 5 \mathrm{mg} / \mathrm{ml}$, and $100 \mathrm{mg} /$ $\mathrm{ml}$, respectively. Dosing was based on pretreatment weights $(1 \mathrm{mg} / \mathrm{kg}$ body weight; ref. 20) in $30 \mu \mathrm{l}$ total PBS volume for daily treatments and in $210 \mu$ l total saline volume for weekly treatments. Mice were injected daily via i.p. injection at $7 \mathrm{am}$. On injection days, stock solutions stored at $-20^{\circ} \mathrm{C}$ were diluted into sterile Eppendorf tubes containing sterile PBS (catalog 14190, Life Technologies). Sterile BD Micro-Fine IV Insulin Syringes (catalog 14-829-1A, Fisher Scientific) were used to inject the i.p. cavity of nonsedated animals. Mice were weighed 3 times per week over the course of 4 weeks at 7 am. Body mass was expressed as fold change from day 0. Cardiotoxin injury was performed injecting $20 \mu \mathrm{l}$ of a $10 \mu \mathrm{M}$ cardiotoxin (discontinued, Sigma-Aldrich) solution in PBS in target muscles in sedated animals (3\% isoflurane, $0.8 \mathrm{l} / \mathrm{min}$ $\mathrm{O}_{2}$ ). Cardiotoxin was released in the center of the muscle through the whole major axis in order to have a homogenous area of injury at the center of the muscle.

Grip strength, treadmill, muscle mechanics, blood drawing, and plethysmography. Forelimb grip strength was monitored using a meter (catalog 1027SM, Columbus Instruments) with the investigator blinded to treatment group. Animals performed 10 pulls with 5 seconds rest on a flat surface between pulls. Mice were run on a treadmill (Exer3/6 without electrical stimulation grills, Columbus Instruments) with a $15^{\circ}$ incline with a start speed of $1 \mathrm{~m} / \mathrm{min}$ with $1 \mathrm{~m} / \mathrm{min}^{2}$ acceleration. The assay was interrupted if the animal stopped for longer than $15 \mathrm{sec}-$ onds on the rest pad. Immediately before sacrifice, in situ tetanic force from tibialis anterior muscle was measured using a Whole Mouse Test System (catalog 1300A, Aurora Scientific) with a $1 \mathrm{~N}$ dual-action lever arm force transducer (300C-LR, Aurora Scientific) in anesthetized animals ( $3 \%$ isoflurane in $100 \% \mathrm{O}_{2}$ ). Tetanic isometric contraction was induced with the following specifications: initial delay, $0.1 \mathrm{sec}-$ onds; frequency, $200 \mathrm{~Hz}$; pulse width, $0.5 \mathrm{msec}$; duration, $0.5 \mathrm{sec}-$ onds; using $100 \mathrm{~mA}$ stimulation (59). Length was adjusted to a fixed baseline of $30 \mathrm{mN}$ resting tension for all muscles/conditions. Fatigue analysis was conducted by repeating tetanic contractions every 10 seconds until complete exhaustion of the muscle (50 cycles). Time of contraction was assessed as time to maximum tetanic value within the 0.0-0.5 second range of each tetanic contraction, while time of relaxation was assessed as time to $90 \%$ minimum tetanic value within the $0.5-0.8$ second range of every tetanus.

Unanesthetized WBP was used to measure respiratory function using a Buxco FinePointe 4-site apparatus (Data Sciences International). Individual mice were placed in a calibrated cylindrical chamber at room temperature. Each mouse was allowed to acclimate to the plethysmography chamber for 120 minutes before recording was initiated. Data were recorded for a total of 15 minutes broken into 3 consecutive 5 -minute periods. All physiological studies were conducted by investigators blinded to treatment group.

Histology, immunofluorescence microscopy, and antibodies. Macrophage detection used anti-F4/80 conjugated to Alexa Fluor 488 (catalog ab6640, Abcam) at a dilution of $1: 100$ overnight at $4^{\circ} \mathrm{C}$; nuclei were counterstained with $0.5 \mu \mathrm{g} / \mathrm{ml}$ Hoechst PBS (45 minutes, room temperature). For fiber typing, sections were incubated with primary antibodies BA-F8 (1:10), SC-71 (1:30), and BF-F3 (1:10, all by Developmental Studies Hybridoma Bank) overnight at $4^{\circ} \mathrm{C}$. Then sections were incubated with secondary antibodies Alexa Fluor 350 anti-IgG2b, Alexa Fluor 488 anti-IgG1, and Alexa Fluor 594 anti-IgM (catalog A21140, A21121, 1010111, Life Technologies). Type 1 fibers were stained blue, type 2A stained green, and type 2B stained red (60). 
Imaging was performed with a Zeiss Axio Observer A1 microscope, using $10 \times$ and $20 \times$ objectives. Brightfield pictures were acquired via Gryphax software (version 1.0.6.598, Jenoptik), while immunofluorescence pictures were acquired via ZEN 2 software (version 2011, Zeiss). Area quantitation, length measurement, and cell counting were performed by means of Image J (NIH).

Hydroxyproline quantification. Hydroxyproline content was measured on $100 \mathrm{mg}$ portions of frozen quadriceps muscles as previously described (61). Results were reported as nM hydroxyproline/mg (tissue).

Serum collection and CK analysis. After completion of the physiologic analyses, serum was acquired and processed as previously described (19). Serum CK was analyzed in triplicate for each mouse using the EnzyChrom Creatine Kinase Assay (catalog ECPK-100, BioAssay Systems) following the manufacturer's instructions. Results were acquired with the Synergy HTX Multi-Mode Plate Reader (BioTek). Results are expressed as $\mathrm{U} / \mathrm{ml}$.

Transcription factor-binding site prediction. Prediction of transcription factor-binding sites was conducted by interrogating genomic sequences of query genes (UCSC, mm 10 assembly, including $10 \mathrm{~kb}$ upstream of TSS) for predicted binding sequences as reported by the JASPAR database and described previously (62). Alignments were conducted in both strand directions with ApE software (v2.0.47, M.W. Davis, University of Utah, Salt Lake City, Utah, USA). One mismatch in noncritical positions, i.e., less than $95 \%$ conserved, was allowed. Prediction of miRNA-binding sites was conducted using the microRNA.org database (63).

Quantitative RT-PCR. Gene expression analysis was conducted on total RNA extracted with TRIzol (catalog 15596018, Life Technologies) from $30 \mathrm{mg}$ tissue per the manufacturer's instructions. RNA from the myofiber fraction was obtained after separating muscle tissue in myofiber- and mononuclear-enriched fractions as follows. Whole muscle (tibialis anterior) was cut in approximately 2-mm-thick myofiber bundles, following the natural myofiber orientation. Bundles were then incubated in collagenase II and gently mechanically dissociated, following the conditions used for myofiber isolation from FDB muscles (as detailed above). The mononuclear-enriched fraction was filtered out through a $40-\mu \mathrm{m}$ cell strainer (catalog 22363547, Fisher Scientific), while the myofiber-enriched fraction was recovered as the unfiltered fraction and further processed with TRIzol. Efficient separation of the 2 fractions was confirmed via microscopy and qPCR of dedicated distinctive markers, and $2 \mu \mathrm{g}$ of RNA was reverse transcribed by means of the qScript cDNA Kit (catalog 95048, Quanta Biosciences) following the manufacturer's instructions. cDNA was diluted 1:7, and $2 \mu \mathrm{l}$ was used per $10 \mu \mathrm{l}$ qPCR reaction. Each qPCR reaction contained 100 $\mathrm{nM}$ primers and $5 \mu \mathrm{l}$ iTaq SYBR Green Mix (catalog 1725124, Bio-Rad). A detailed list of primers and sequences is provided in Supplemental Table 3. Fluorescence was quantitated using the CFX96 Real-Time System (Bio-Rad). miR-16 was used as internal normalizer.

miRNA expression studies. miRNA expression analyses were performed from total RNA from tissue. miRNAs were reverse transcribed by means of the TaqMan MicroRNA Reverse Transcription Kit (cata$\log 4366596$, Life Technologies) following the manufacturer's protocol and using $4 \mu \mathrm{g}$ of total tissue RNA. cDNA was diluted 1:15, and 2 $\mu \mathrm{l}$ was used per $10 \mu \mathrm{l}$ qPCR reaction. Each qPCR reaction contained $100 \mathrm{nM}$ primers and $5 \mu$ l of TaqMan Master Mix (catalog 4427788, Life Technologies). Both reverse transcription and qPCR reactions were conducted using miRNA-specific TaqMan Assays (catalog 4427975,
Life Technologies). Fluorescence was quantitated using the CFX96 Real-Time System (Bio-Rad). miR-16 was used as internal control.

ChIP-qPCR and luciferase assays. ChIP-qPCR was performed according to a previously reported protocol (64) and adjusted conditions (59). Results were expressed as percentages of raw expression of the respective input. ChIP-qPCR assays on isolated myofibers were performed as above, with the following steps before chromatin sonication. Freshly isolated whole gastrocnemius muscles were finely minced and digested in $5 \mathrm{ml} / \mathrm{muscle}$ of PBS supplemented with $1 \mathrm{mM}$ $\mathrm{CaCl}_{2}$ and $100 \mathrm{U} / \mathrm{ml}$ collagenase II (catalog 17101, Life Technologies) at $37^{\circ} \mathrm{C}$ for 1 hour with shaking. The suspension was then filtered through a $40-\mu \mathrm{m}$ strainer (catalog 22363547, Fisher Scientific) and the unfiltered fraction (enriched in myofibers) was kept for further steps. Separation of mononuclear fraction in the filtered fraction was confirmed at the microscope. Myofibers were lysed in lysis buffer, using $700 \mu \mathrm{l}$ per muscle, with approximately $250 \mu \mathrm{l} 2.3-\mathrm{mm}$ zirconia/silica beads (catalog 11079125z, BioSpec). Lysis buffer consisted of $10 \mathrm{mM}$ HEPES (pH 7.3, catalog H3375), $10 \mathrm{mM} \mathrm{KCl} \mathrm{(catalog} \mathrm{P9541),} 5 \mathrm{mM}$ $\mathrm{MgCl}_{2}$ (catalog M8266), $0.5 \mathrm{mM}$ DTT (catalog 646563), and $3 \mu \mathrm{g} / \mathrm{ml}$ cytochalasin B (C6762, all reagents from Sigma-Aldrich) and protease inhibitor cocktail (catalog 11852700, Roche). Myofibers were them homogenized by means of Mini-BeadBeater-16 (catalog 607, Biospec) for 30 seconds, then by rotating at $4^{\circ} \mathrm{C}$ for 30 minutes. Samples were centrifuged at $3,000 \mathrm{~g}$ for 5 minutes at $4^{\circ} \mathrm{C}$; supernatant was removed. The pellet was resuspended in lysis buffer supplemented with $3 \mu \mathrm{g} / \mathrm{ml}$ cytochalasin B, as per reported conditions (64), and incubated on ice for 10 minutes. Nuclei were pelleted at $300 \mathrm{~g}$ for 10 minutes at $4^{\circ} \mathrm{C}$ and resuspended in $1 \mathrm{ml}$ 1\% PFA for 5 minutes at room temperature. Fixation was quenched with $100 \mu \mathrm{l}$ of $1.375 \mathrm{M}$ glycine (catalog BP381-5, Fisher Scientific). Nuclei were repelleted as before and subsequently processed following reported protocol (64) and the procedures mentioned for myoblasts, with the adjustment of adding $3 \mu \mathrm{g} / \mathrm{ml}$ cytochalasin B into all solutions for chromatin preparation and sonication, antibody incubation, and wash steps.

Ex vivo luciferase assay was performed on whole, electroporated FDB muscles. Muscles were minced and homogenized in the lysate buffer, and experiments were performed according to the Dual Luciferase Assay Kit (catalog 1910, Promega) instructions. Luminescence was recorded using the Synergy HTX Multi-Mode 96-Well Plate Reader (BioTek). Raw values were normalized to Renilla luciferase, then to protein content (MyHC), and finally either to empty pGL4.23 vector (unregulated negative control) and to vehicle-treated muscles with the same plasmids (Figure 3) or to pMiR empty vector-transfected muscles (unregulated, positive control) (Figure 4). For promoter site analysis, raw values were normalized to Renilla luciferase, protein content (MyHC), and empty pGL4.23-transfected muscles. Results are expressed as fold change to average vehicle.

RNA-seq analysis. A detailed explanation of RNA-seq protocols and analyses can be found in Supplemental Methods. All original microarray data were deposited in the NCBI's Gene Expression Omnibus (65) (GEO GSE95682).

Primary myoblast isolation. Primary myoblasts were sorted as $\mathrm{CD} 6^{+}$cells from the mononuclear preparation obtained from tibialis anterior muscles, adapting previously reported conditions (22).

Statistics. Statistical analyses were performed with Prism (GraphPad). Most comparisons relied on ANOVA with Bonferroni's multicomparison appropriate for the variables tested (1-way ANOVA for 1 
variable, 2-way ANOVA for 2 variables, typically time and treatment). When comparing 2 groups, 2-tailed Student's $t$ test with Welch's correction (unequal variances) was used. For ANOVA and $t$ test analyses, a $P$ value of less than 0.05 was considered significant. Data were presented as single values (dot plots, histograms) when the number of data points was less than 10. In analyses pooling larger data point sets per group, Tukey distribution bars were used to emphasize data range distribution, and histograms with error bars were used to emphasize shifts in average values. Analysis pooling data points over time was presented as marked line plots. Dot plots, histograms, and marked line plots depict mean \pm SEM. Box plots depict the Tukey distribution of the data pool: interquartile distribution; lower whisker, 25 th percentile minus 1.5 times the interquartile range; upper whisker, 75 th percentile plus 1.5 times the interquartile range.

Study approval. The study was conducted with the approval of Northwestern University's Institutional Animal Care and Use Committee.

\section{Author contributions}

MQ conducted experiments and drafted the manuscript. DYB conducted experiments and analysis. JLW performed experiments and analysis. AHV conducted analysis. MH assisted with experiments. JUE performed experiments. ARD conducted experiments and analysis. EMM conceived the study, analyzed data, and edited the manuscript.

\section{Acknowledgments}

This work was supported by NIH U54 AR052646, NIH RO1 NS047726, the Parent Project for Muscular Dystrophy, and the American Heart Association. MQ is supported by the Muscular Dystrophy Association and the American Association of Neuromuscular \& Electrodiagnostic Medicine (AANEM) Foundation for Research \& Education (development grant 479350). We acknowledge the outstanding support from the Center for Advanced Microscopy and Constadina Arvanitis and Joshua Rappoport at Northwestern University. Cell sorting was conducted at the Flow Cytometry Core Facility of Northwestern University, supported by a Cancer Center support grant (NCI CA060553) and the NIH (1S10OD011996-01). RNA sequencing was conducted at the NUSeq Core Facility (Center for Genetic Medicine, Northwestern University).

Address correspondence to: Elizabeth M. McNally, Center for Genetic Medicine, Northwestern University, 303 E. Superior Lurie 7-123, Chicago, Illinois 60611, USA. Phone: 312.503.6258; E-mail: elizabeth.mcnally@northwestern.edu.
1. Kanda F, Okuda S, Matsushita T, Takatani K, Kimura KI, Chihara K. Steroid myopathy: pathogenesis and effects of growth hormone and insulin-like growth factor-I administration. Horm Res. 2001;56(Suppl 1):24-28.

2. Bello L, et al. Prednisone/prednisolone and deflazacort regimens in the CINRG Duchenne Natural History Study. Neurology. 2015;85(12):1048-1055.

3. Matthews E, Brassington R, Kuntzer T, Jichi F, Manzur AY. Corticosteroids for the treatment of Duchenne muscular dystrophy. Cochrane Database Syst Rev. 2016;(5):CD003725.

4. Wehling-Henricks M, Lee JJ, Tidball JG. Prednisolone decreases cellular adhesion molecules required for inflammatory cell infiltration in dystrophin-deficient skeletal muscle. Neuromuscul Disord. 2004;14(8-9):483-490.

5. Fisher I, et al. Prednisolone-induced changes in dystrophic skeletal muscle. FASEB J. 2005;19(7):834-836.

6. Peverelli L, et al. Histologic muscular history in steroid-treated and untreated patients with Duchenne dystrophy. Neurology. 2015;85(21):1886-1893.

7. Anderson JE, McIntosh LM, Poettcker R. Deflazacort but not prednisone improves both muscle repair and fiber growth in diaphragm and limb muscle in vivo in the $\mathrm{mdx}$ dystrophic mouse. Muscle Nerve. 1996;19(12):1576-1585.

8. St-Pierre SJ, Chakkalakal JV, Kolodziejczyk SM, Knudson JC, Jasmin BJ, Megeney LA. Glucocorticoid treatment alleviates dystrophic myofiber pathology by activation of the calcineurin/NF-AT pathway. FASEB J. 2004;18(15):1937-1939.

9. Baltgalvis KA, Call JA, Nikas JB, Lowe DA. Effects of prednisolone on skeletal muscle contractility in mdx mice. Muscle Nerve. 2009;40(3):443-454.

10. Guerron $\mathrm{AD}$, et al. Functional and molecular effects of arginine butyrate and prednisone on muscle and heart in the mdx mouse model of Duchenne muscular dystrophy. PLOS ONE. 2010;5(6):e11220.

11. Morrison-Nozik A, et al. Glucocorticoids enhance muscle endurance and ameliorate Duchenne muscular dystrophy through a defined metabolic program. Proc Natl Acad Sci U S A. 2015;112(49):E6780-E6789.

12. Griggs RC, et al. Efficacy and safety of deflazacort vs prednisone and placebo for Duchenne muscular dystrophy. Neurology. 2016;87(20):2123-2131.

13. Petrof BJ. Molecular pathophysiology of myofiber injury in deficiencies of the dystrophin-glycoprotein complex. Am J Phys Med Rehabil. 2002;81(11 Suppl):S162-S174.

14. Swaggart KA, et al. Annexin A6 modifies muscular dystrophy by mediating sarcolemmal repair. Proc Natl Acad Sci U S A. 2014;111(16):6004-6009.

15. Roostalu U, Strähle U. In vivo imaging of molecular interactions at damaged sarcolemma. Dev Cell. 2012;22(3):515-529.

16. Demonbreun AR, Quattrocelli M, Barefield DY, Allen MV, Swanson KE, McNally EM. An actin-dependent annexin complex mediates plasma membrane repair in muscle. J Cell Biol. 2016;213(6):705-718.

17. Perretti M, D'Acquisto F. Annexin A1 and glucocorticoids as effectors of the resolution of inflammation. Nat Rev Immunol. 2009;9(1):62-70.

18. Coméra C, Russo-Marie F. Glucocorticoidinduced annexin 1 secretion by monocytes and peritoneal leukocytes. Br J Pharmacol. 1995;115(6):1043-1047.

19. Demonbreun AR, et al. Enhanced muscular dystrophy from loss of dysferlin is accompanied by impaired annexin A6 translocation after sarcolemmal disruption. Am JPathol. 2016;186(6):1610-1622.

20. Sali A, et al. Glucocorticoid-treated mice are an inappropriate positive control for long-term preclinical studies in the $\mathrm{mdx}$ mouse. PLOS ONE. 2012;7(4):e34204.

21. Ambrose MP, Hunninghake GW. Corticosteroids increase lipocortin I in alveolar epithelial cells. Am J Respir Cell Mol Biol. 1990;3(4):349-353.

22. Agley CC, Rowlerson AM, Velloso CP, Lazarus $\mathrm{NL}$, Harridge SD. Isolation quantitative immunocytochemical characterization of primary myogenic cells fibroblasts from human skeletal muscle. J Vis Exp. 2015;(95):52049.

23. Kuo T, Lew MJ, Mayba O, Harris CA, Speed TP, Wang JC. Genome-wide analysis of glucocorticoid receptor-binding sites in myotubes identifies gene networks modulating insulin signaling. Proc Natl Acad Sci U S A. 2012;109(28):11160-11165.

24. Allen DL, Unterman TG. Regulation of myostatin expression and myoblast differentiation by FoxO and SMAD transcription factors. Am J Physiol, Cell Physiol. 2007;292(1):C188-C199.

25. Shin DJ, Joshi P, Hong SH, Mosure K, Shin DG, Osborne TF. Genome-wide analysis of FoxO1 binding in hepatic chromatin: potential involvement of FoxO1 in linking retinoid signaling to hepatic gluconeogenesis. Nucleic Acids Res. 2012;40(22):11499-11509.

26. Gummow BM, Scheys JO, Cancelli VR, Hammer GD. Reciprocal regulation of a glucocorticoid receptor-steroidogenic factor-1 transcription complex on the Dax-1 promoter by glucocorticoids and adrenocorticotropic hormone in the adrenal cortex. Mol Endocrinol. 2006;20(11):2711-2723.

27. Hardy D, et al. Comparative study of injury models for studying muscle regeneration in mice. PLOS ONE. 2016;11(1):e0147198.

28. Schakman O, Gilson H, Kalista S, Thissen JP. Mechanisms of muscle atrophy induced by glucocorticoids. Horm Res. 2009;72(Suppl 1):36-41.

29. Lewis MI, Bodine SC, Kamangar N, Xu X, Da X, Fournier M. Effect of severe short-term malnutri- 
tion on diaphragm muscle signal transduction pathways influencing protein turnover. J Appl Physiol. 2006;100(6):1799-1806.

30. Bodine SC, et al. Identification of ubiquitin ligases required for skeletal muscle atrophy. Science. 2001;294(5547):1704-1708.

31. Sandri M, et al. Foxo transcription factors induce the atrophy-related ubiquitin ligase atrogin-1 and cause skeletal muscle atrophy. Cell. 2004;117(3):399-412.

32. Sasse SK, et al. The glucocorticoid receptor and KLF15 regulate gene expression dynamics and integrate signals through feed-forward circuitry. Mol Cell Biol. 2013;33(11):2104-2115.

33. Schäcke H, Döcke WD, Asadullah K. Mechanisms involved in the side effects of glucocorticoids. Pharmacol Ther. 2002;96(1):23-43.

34. Escolar DM, et al. Randomized, blinded trial of weekend vs daily prednisone in Duchenne muscular dystrophy. Neurology. 2011;77(5):444-452.

35. Keeling RM, Golumbek PT, Streif EM, Connolly AM. Weekly oral prednisolone improves survival and strength in male $\mathrm{mdx}$ mice. Muscle Nerve. 2007;35(1):43-48.

36. Coley WD, et al. Effect of genetic background on the dystrophic phenotype in mdx mice. Hum Mol Genet. 2016;25(1):130-145.

37. Leite PE, et al. Nicotinic acetylcholine receptor activation reduces skeletal muscle inflammation of mdx mice. JNeuroimmunol. 2010;227(1-2):44-51.

38. Winterberg PD, Jiang R, Maxwell JT, Wang B, Wagner MB. Myocardial dysfunction occurs prior to changes in ventricular geometry in mice with chronic kidney disease (CKD). Physiol Rep. 2016;4(5): e12732.

39. Zentner GE, Tesar PJ, Scacheri PC. Epigenetic signatures distinguish multiple classes of enhancers with distinct cellular functions. Genome Res. 2011;21(8):1273-1283.

40. Milan G, et al. Regulation of autophagy and the ubiquitin-proteasome system by the FoxO transcriptional network during muscle atrophy. Nat Commun. 2015;6:6670.

41. McPherron AC, Lawler AM, Lee SJ. Regulation of skeletal muscle mass in mice by a new TGF-beta superfamily member. Nature.
1997;387(6628):83-90.

42. McNeil PL, Steinhardt RA. Loss, restoration, and maintenance of plasma membrane integrity. JCell Biol. 1997;137(1):1-4.

43. Parente L. Deflazacort: therapeutic index, relative potency and equivalent doses versus other corticosteroids. BMC Pharmacol Toxicol. 2017;18(1):1.

44. Cai C, et al. Membrane repair defects in muscular dystrophy are linked to altered interaction between MG53, caveolin-3, and dysferlin. J Biol Chem. 2009;284(23):15894-15902.

45. Waddell LB, et al. Dysferlin, annexin A1, and mitsugumin 53 are upregulated in muscular dystrophy and localize to longitudinal tubules of the T-system with stretch. J Neuropathol Exp Neurol. 2011;70(4):302-313.

46. Van Laethem F, et al. Glucocorticoids alter the lipid and protein composition of membrane rafts of a murine $\mathrm{T}$ cell hybridoma. J Immunol. 2003;170(6):2932-2939.

47. Markham LW, Spicer RL, Khoury PR, Wong BL, Mathews KD, Cripe LH. Steroid therapy and cardiac function in Duchenne muscular dystrophy. Pediatr Cardiol. 2005;26(6):768-771.

48. Janssen PM, et al. Prednisolone attenuates improvement of cardiac and skeletal contractile function and histopathology by lisinopril and spironolactone in the $\mathrm{mdx}$ mouse model of Duchenne muscular dystrophy. PLoS ONE. 2014;9(2):e88360.

49. Haldar SM, et al. Kruppel-like factor 15 regulates skeletal muscle lipid flux and exercise adaptation. Proc Natl Acad Sci U S A. 2012;109(17):6739-6744.

50. Zhang L, et al. KLF15 establishes the landscape of diurnal expression in the heart. Cell Rep. 2015;13(11):2368-2375.

51. Gong S, et al. Dynamics and correlation of serum cortisol and corticosterone under different physiological or stressful conditions in mice. PLOS ONE. 2015;10(2):e0117503.

52. Karlamangla AS, Friedman EM, Seeman TE, Stawksi RS, Almeida DM. Daytime trajectories of cortisol: demographic and socioeconomic differences--findings from the National Study of Daily Experiences. Psychoneuroendocrinology. 2013;38(11):2585-2597.
53. Asada M, et al. DNA binding-dependent glucocorticoid receptor activity promotes adipogenesis via Krüppel-like factor 15 gene expression. $L a b$ Invest. 2011;91(2):203-215.

54. Masuno K, et al. Expression profiling identifies Klf15 as a glucocorticoid target that regulates airway hyperresponsiveness. Am J Respir Cell Mol Biol. 2011;45(3):642-649.

55. Shimizu N, et al. Crosstalk between glucocorticoid receptor and nutritional sensor mTOR in skeletal muscle. Cell Metab. 2011;13(2):170-182.

56. Shimizu N, et al. A muscle-liver-fat signalling axis is essential for central control of adaptive adipose remodelling. Nat Commun. 2015;6:6693.

57. DiFranco M, Quinonez M, Capote J, Vergara J. DNA transfection of mammalian skeletal muscles using in vivo electroporation. J Vis Exp. 2009;(32):1520.

58. Demonbreun AR, McNally EM. DNA electroporation, isolation imaging of myofibers. J Vis Exp. 2015;(106):e53551.

59. Quattrocelli M, et al. Mesodermal iPSCderived progenitor cells functionally regenerate cardiac and skeletal muscle. JClin Invest. 2015;125(12):4463-4482.

60. Bloemberg D, Quadrilatero J. Rapid determination of myosin heavy chain expression in rat, mouse, and human skeletal muscle using multicolor immunofluorescence analysis. PLOS ONE. 2012;7(4):e35273.

61. Heydemann A, et al. Latent TGF-beta-binding protein 4 modifies muscular dystrophy in mice. JClin Invest. 2009;119(12):3703-3712.

62. Jolma A, et al. DNA-binding specificities of human transcription factors. Cell. 2013;152(1-2):327-339.

63. Betel D, Wilson M, Gabow A, Marks DS, Sander C. The microRNA.org resource: targets and expression. Nucleic Acids Res. 2008; 36(Database issue):D149-D153.

64. Carey MF, Peterson CL, Smale ST. Chromatin immunoprecipitation (ChIP). Cold Spring Harb Protoc. 2009;2009(9):pdb.prot5279.

65. Edgar R, Domrachev M, Lash AE. Gene Expression Omnibus: NCBI gene expression and hybridization array data repository. Nucleic Acids Res. 2002;30(1):207-210. 